\title{
Strong Couplings of Three Mesons with Charm(ing) Involvement
}

\author{
Wolfgang Lucha ${ }^{1, a}$, Dmitri Melikhov ${ }^{1,2,3, b}$, Hagop Sazdjian ${ }^{4, c}$, and Silvano Simula ${ }^{5, d}$ \\ ${ }^{1}$ Institute for High Energy Physics, Austrian Academy of Sciences, Nikolsdorfergasse 18, A-1050 Vienna, \\ Austria \\ ${ }^{2}$ D. V. Skobeltsyn Institute of Nuclear Physics, M. V. Lomonosov Moscow State University, 119991, Moscow, \\ Russia \\ ${ }^{3}$ Faculty of Physics, University of Vienna, Boltzmanngasse 5, A-1090 Vienna, Austria \\ ${ }^{4}$ IPN, CNRS/IN2P3, Université Paris-Sud 11, F-91406 Orsay, France \\ 5 INFN, Sezione di Roma Tre, Via della Vasca Navale 84, I-00146 Roma, Italy
}

\begin{abstract}
We determine the strong couplings of three mesons that involve, at least, one $\eta_{c}$ or $J / \psi$ meson, within the framework of a constituent-quark model by means of relativistic dispersion formulations. For strong couplings of $J / \psi$ mesons to two charmed mesons, our approach leads to predictions roughly twice as large as those arising from QCD sum rules.
\end{abstract}

\section{Three-meson strong coupling from meson-meson transition amplitudes}

We determine the strong couplings of three mesons at least one of which is one of the charmonia $\eta_{c}$ and $J / \psi$, generically called $g_{P P^{\prime} V}$ and $g_{P V^{\prime} V}$ for pseudoscalar mesons $P$ of mass $M_{P}$ and vector mesons $V$ of mass $M_{V}$ and polarization vector $\varepsilon_{\mu}$ and defined, for momentum transfer $q \equiv p_{1}-p_{2}$, by the amplitudes

$$
\begin{aligned}
& \left\langle P^{\prime}\left(p_{2}\right) V(q) \mid P\left(p_{1}\right)\right\rangle=-\frac{g_{P P^{\prime} V}}{2}\left(p_{1}+p_{2}\right)^{\mu} \varepsilon_{\mu}^{*}(q), \\
& \left\langle V^{\prime}\left(p_{2}\right) V(q) \mid P\left(p_{1}\right)\right\rangle=-g_{P V^{\prime} V} \epsilon_{\mu \nu \rho \sigma} \varepsilon^{* \mu}(q) \varepsilon^{* v}\left(p_{2}\right) p_{1}^{\rho} p_{2}^{\sigma},
\end{aligned}
$$

from the residues of poles situated at the masses $M_{P_{R}}$ and $M_{V_{R}}$ of (appropriate) pseudoscalar and vector resonances $P_{R}$ and $V_{R}$ and contributing to transition form factors $F_{+}^{P>P^{\prime}}\left(q^{2}\right), V^{P>V}\left(q^{2}\right)$ and $A_{0}^{P>V}\left(q^{2}\right)$, in terms of vector quark currents $j_{\mu} \equiv \bar{q}_{1} \gamma_{\mu} q_{2}$ and axial-vector quark currents $j_{\mu}^{5} \equiv \bar{q}_{1} \gamma_{\mu} \gamma_{5} q_{2}$ defined by

$$
\begin{array}{ll}
\left\langle P^{\prime}\left(p_{2}\right)\left|j_{\mu}\right| P\left(p_{1}\right)\right\rangle=F_{+}^{P>P^{\prime}}\left(q^{2}\right)\left(p_{1}+p_{2}\right)_{\mu}+\cdots, & \left.F_{+}^{P>P^{\prime}}\left(q^{2}\right)\right|_{\text {pole }}=\frac{g_{P P^{\prime} V_{R}} f_{V_{R}}}{2 M_{V_{R}}\left(1-q^{2} / M_{V_{R}}^{2}\right)}, \\
\left\langle V\left(p_{2}\right)\left|j_{\mu}\right| P\left(p_{1}\right)\right\rangle=\frac{2 V^{P>V}\left(q^{2}\right)}{M_{P}+M_{V}} \epsilon_{\mu \nu \rho \sigma} \varepsilon^{* v}\left(p_{2}\right) p_{1}^{\rho} p_{2}^{\sigma}, & \left.V^{P>V}\left(q^{2}\right)\right|_{\text {pole }}=\frac{\left(M_{V}+M_{P}\right) g_{P V V_{R}} f_{V_{R}}}{2 M_{V_{R}}\left(1-q^{2} / M_{V_{R}}^{2}\right)}, \\
\left\langle V\left(p_{2}\right)\left|j_{\mu}^{5}\right| P\left(p_{1}\right)\right\rangle=\mathrm{i} q_{\mu}\left(\varepsilon^{*}\left(p_{2}\right) p_{1}\right) \frac{2 M_{V}}{q^{2}} A_{0}^{P>V}\left(q^{2}\right)+\cdots, & \left.A_{0}^{P>V}\left(q^{2}\right)\right|_{\text {pole }}=\frac{g_{P P_{R} V} f_{P_{R}}}{2 M_{V}\left(1-q^{2} / M_{P_{R}}^{2}\right)},
\end{array}
$$

\footnotetext{
a e-mail: Wolfgang.Lucha@oeaw.ac.at

be-mail: dmitri_melikhov@gmx.de

ce-mail: sazdjian@ipno.in2p3.fr

$\mathrm{d}_{\mathrm{e}-\mathrm{mail}}$ simula@roma3.infn.it
} 
where the $P$ or $V$ decay constants $f_{P, V}$ parametrize the matrix elements of the interpolating currents $j_{\mu}^{(5)}$

$$
\left\langle 0\left|j_{\mu}^{5}\right| P(q)\right\rangle=\mathrm{i} f_{P} q_{\mu}, \quad\left\langle 0\left|j_{\mu}\right| V(q)\right\rangle=f_{V} M_{V} \varepsilon_{\mu}(q) .
$$

Such strong-coupling results may prove to be useful for studies of long-distance QCD effects in hadron decays involving charmed mesons or charmonia in the final state of a kind similar to the one in Ref. [1].

\section{Quark-model-underpinned dispersion analysis of transition form factors}

We describe the relevant properties of the involved strongly coupling mesons by means of a relativistic constituent-quark model [2-4]. Of course, this requires us to match the QCD currents $j_{\mu}^{(5)}$ to associated constituent-quark currents, which is, for heavy quarks, easily effected by introducing form factors $g_{V, A}$,

$$
j_{\mu}=g_{V} \bar{Q}_{1} \gamma_{\mu} Q_{2}+\text { other Lorentz structures , } \quad j_{\mu}^{5}=g_{A} \bar{Q}_{1} \gamma_{\mu} \gamma_{5} Q_{2}+\text { other Lorentz structures , }
$$

for which we choose $g_{V}=g_{A}=1$ [5] but, for light quarks, rendered rather involved [6, 7], for instance, if embedding partial axial-current conservation. For the radial meson wave functions, Gaussian shapes

$$
w_{P, V}\left(k^{2}\right) \propto \exp \left(-\frac{k^{2}}{2 \beta_{P, V}^{2}}\right), \quad \int \mathrm{d} k k^{2} w_{P, V}^{2}\left(k^{2}\right)=1,
$$

with slopes $\beta_{P, V}$ given, together with all relevant mesonic features, in Table 1 [8-13], turn out to suffice for our purposes. Table 2 lists the numerical values adopted for the masses of the constituent quarks $Q$. Within the framework of a relativistic dispersion formalism (reviewed, e.g., in Ref. [14]), we represent each transition form factor $\mathcal{F}\left(q^{2}\right)=F_{+}^{P>P^{\prime}}\left(q^{2}\right), V^{P>V}\left(q^{2}\right), A_{0}^{P>V}\left(q^{2}\right)$ by a double dispersion integral of a double spectral density $\Delta_{\mathcal{F}}\left(s_{1}, s_{2}, q^{2}\right)$ the one-loop contributions to which derive from Feynman graphs like the ones in Fig. 1 and each decay constant $f_{P, V}$ by a dispersion integral of a spectral density $\rho_{P, V}(s)$,

$$
\mathcal{F}\left(q^{2}\right)=\int \mathrm{d} s_{1} \mathrm{~d} s_{2} \phi_{1}\left(s_{1}\right) \phi_{2}\left(s_{2}\right) \Delta_{\mathcal{F}}\left(s_{1}, s_{2}, q^{2}\right), \quad f_{P, V}=\int_{\left(m_{1}+m\right)^{2}}^{\infty} \mathrm{d} s \phi_{P, V}(s) \rho_{P, V}(s),
$$

Table 1. Relevant parameters of the mesons: numerical values of mass $M$, leptonic decay constant $f$ and slope $\beta$.

\begin{tabular}{lcll}
\hline Meson & $M(\mathrm{GeV})$ & $f(\mathrm{MeV})$ & $\beta(\mathrm{GeV})$ \\
\hline$D$ & 1.87 & $206 \pm 8$ & 0.475 \\
$D^{*}$ & 2.010 & $260 \pm 10$ & 0.48 \\
$D_{s}$ & 1.97 & $248 \pm 2.5$ & 0.545 \\
$D_{s}^{*}$ & 2.11 & $311 \pm 9$ & 0.54 \\
$\eta_{c}$ & 2.980 & $394.7 \pm 2.4$ & 0.77 \\
$J / \psi$ & 3.097 & $405 \pm 7$ & 0.68 \\
\hline
\end{tabular}

Table 2. Constituent mass of each quark flavour $Q=u, d, s, c[5]$ involved in charm(ing) three-meson couplings.

\begin{tabular}{lc}
\hline Quark flavour & Quark mass $m(\mathrm{GeV})$ \\
\hline$u$ & 0.23 \\
$d$ & 0.23 \\
$s$ & 0.35 \\
$c$ & 1.45 \\
\hline
\end{tabular}



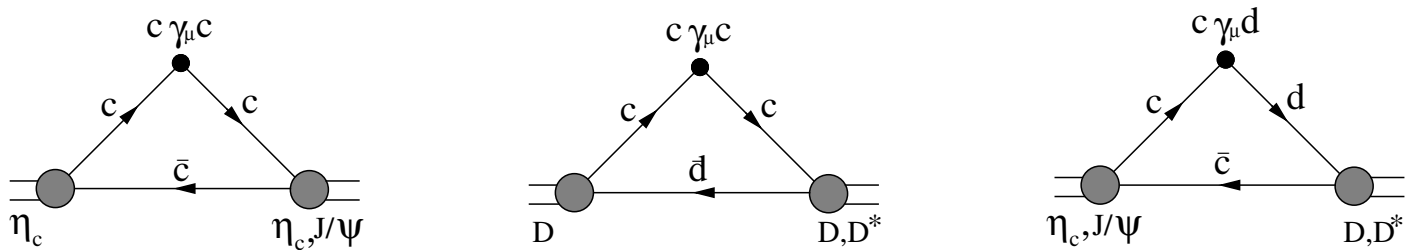

Figure 1. Feynman graphs of transitions among the mesons $\eta_{c}, J / \psi, D, D^{*}$, induced by the currents $\bar{c} \gamma_{\mu} c$ or $\bar{c} \gamma_{\mu} d$.

and of the wave functions of all mesons entering the corresponding one- or two-meson matrix elements

$$
\phi_{P, V}(s)=\frac{\pi}{s^{3 / 4}} \sqrt{\frac{s^{2}-\left(m_{1}^{2}-m^{2}\right)^{2}}{2\left[s-\left(m_{1}-m\right)^{2}\right]}} w_{P, V}\left(\frac{\left(s-m_{1}^{2}-m^{2}\right)^{2}-4 m_{1}^{2} m^{2}}{4 s}\right) .
$$

\section{Three-meson strong coupling: determination from transition amplitudes}

We fix the slopes $\beta_{P, V}$ such that the decay constants $f_{P, V}$ are reproduced by their spectral representation. Equipped with these $\beta_{P, V}$ values, we deduce all strong couplings from the spectral representation of the relevant form factors $\mathcal{F}\left(q^{2}\right)$ derived sufficiently off the resonances at $M_{R}\left(R=P_{R}, V_{R}\right)$, by interpolating pointwise given momentum dependences of $\mathcal{F}\left(q^{2}\right)$ by three-parameter $\left(\sigma_{1,2}, \mathcal{F}(0)\right)$ ansätze of the form

$\mathcal{F}\left(q^{2}\right)=\frac{\mathcal{F}(0)}{1-\sigma_{1} q^{2} / M_{R}^{2}+\sigma_{2} q^{4} / M_{R}^{4}} \frac{1}{1-q^{2} / M_{R}^{2}}, \quad \operatorname{Res} \mathcal{F}\left(M_{R}^{2}\right)=\frac{\mathcal{F}(0)}{1-\sigma_{1}+\sigma_{2}}, \quad R=P_{R}, V_{R}$,

and extrapolating $\mathcal{F}\left(q^{2}\right)$ to the poles at $q^{2}=M_{R}^{2}$, where the strong couplings emerge from the residues. Using $\sigma_{1,2}, \mathcal{F}(0)$, and $M_{R}$ as fit parameters, all arising masses $M_{R}$ come close to the known resonances. Quite generally, a given strong coupling may show up in and therefore can be extracted from more than one meson-meson transition form factor, for example, $g_{\eta_{c} \eta_{c} \psi}$ from $F_{+}^{\eta_{c}>\eta_{c}}$ or $A_{0}^{\eta_{c}>\psi}$ (see Fig. 2) [15-17], $g_{D D \psi}$ from $F_{+}^{D>D}$ or $A_{0}^{D>\psi}$ (see Fig. 3(a)) [15-17] and $g_{D D^{*}} \eta_{c}$ from $F_{+}^{\eta_{c}>D}, A_{0}^{\eta_{c}>D^{*}}$ or $A_{0}^{D>D^{*}}$ (see Fig. 3(b)) [15-17]; for further examples of such multiple involvements, consult Tables III, V, and VI of Ref. [15].

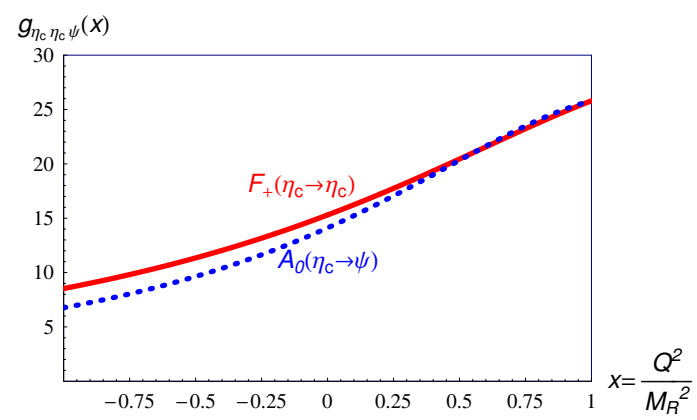

Figure 2. Behaviour of the off-shell $\eta_{c}-\eta_{c}-J / \psi$ strong coupling $g_{\eta_{c} \eta_{c} \psi}$ with increasing resonance-mass-normalized momentum transfer $x \equiv q^{2} / M_{R}^{2}$ for the transition of $\eta_{c}$ to the $\eta_{c}$ (solid red line) or the $J / \psi$ meson (dotted blue line). 


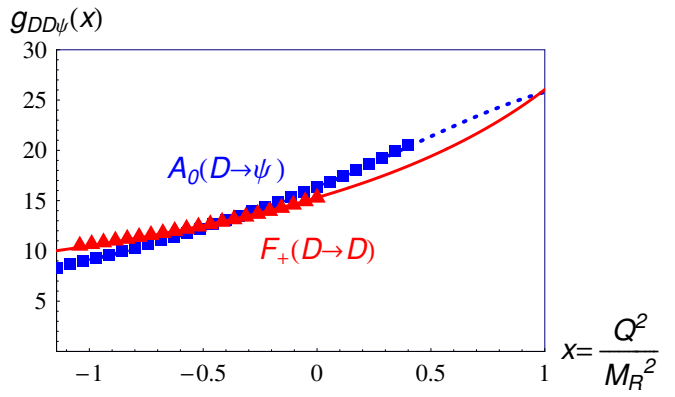

(a)

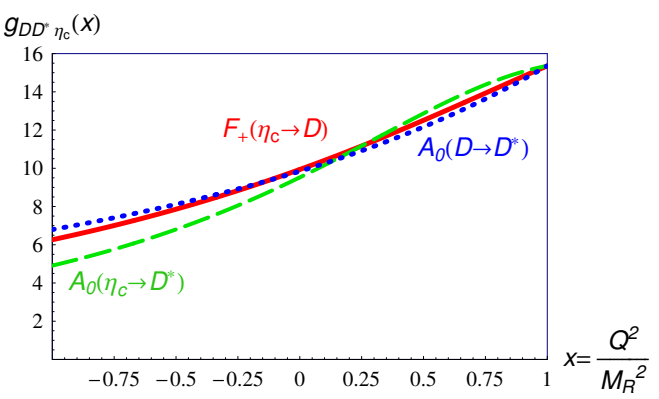

(b)

Figure 3. Behaviour of the "off-shell" $D-D-J / \psi$ and $D-D^{*}-\eta_{c}$ strong couplings $g_{D D \psi}$ and $g_{D D^{*} \eta_{c}}$, respectively, with increasing "resonance-mass-normalized" momentum transfer $x \equiv q^{2} / M_{R}^{2}$ : (a) $g_{D \hat{D} \psi}(x)=2 M_{\psi}(1-x) A_{0}^{D>\psi}\left(q^{2}\right) / f_{D}$ (blue dotted line and squares $\boldsymbol{a}$ ) and $g_{D D \hat{\psi}}(x)=2 M_{\psi}(1-x) F_{+}^{D>D}\left(q^{2}\right) / f_{\psi}$ (red solid line and triangles $\boldsymbol{\Delta}$ ), relying on interpolation (blue or red lines) or not (squares $\mathbf{\square}$, triangles $\mathbf{\Lambda}$ ); (b) $g_{D D^{*} \eta_{c}}(x)$ (solid red line), $g_{D D^{*} \hat{\eta}_{c}}(x)$ (dotted blue line) and $g_{\hat{D} D^{*} \eta_{c}}(x)$ (dashed green line). For each transition, the relevant resonance, $R$, is identified by a circumflex.

\section{Strong coupling predictions from relativistic constituent-quark approach}

We collect our emerging strong-coupling findings - extracted, in the case of multipresence of one and the same three-meson coupling in more than one meson-meson transition amplitude, by a combined fit — in Table 3: Strange quark content instead of a down quark implies a reduction of the involved strong couplings, by roughly $10 \%$. Confronting, in Table 4 , our $D_{(s)}-D_{(s)}^{(*)}-J / \psi$ predictions with QCD sum-rule outcomes [18-20], the QCD sum-rule estimates prove to be lower than ours [15-17] by a factor of two.

Table 3. Charm(ing) three-meson strong couplings: quark-model-based dispersion-approach outcomes [15-17].

\begin{tabular}{lc}
\hline$P P^{\prime} V$ Coupling & Strong coupling $g_{P P^{\prime} V}$ \\
\hline$\eta_{c}-\eta_{c}-J / \psi$ & $25.8 \pm 1.7$ \\
$D-D-J / \psi$ & $26.04 \pm 1.43$ \\
$D-D^{*}-\eta_{c}$ & $15.51 \pm 0.45$ \\
$D_{s}-D_{s}-J / \psi$ & $23.83 \pm 0.78$ \\
$D_{s}-D_{s}^{*}-\eta_{c}$ & $14.15 \pm 0.52$ \\
\hline
\end{tabular}

\begin{tabular}{lc}
\hline$P V^{\prime} V$ coupling & Strong coupling $g_{P V^{\prime} V}\left(\mathrm{GeV}^{-1}\right)$ \\
\hline$\eta_{c^{-}} J / \psi-J / \psi$ & $10.6 \pm 1.5$ \\
$D-D^{*}-J / \psi$ & $10.7 \pm 0.4$ \\
$D^{*}-D^{*}-\eta_{c}$ & $9.76 \pm 0.32$ \\
$D_{s}-D_{s}^{*}-J / \psi$ & $9.6 \pm 0.8$ \\
$D_{s}^{*}-D_{s}^{*}-\eta_{c}$ & $8.27 \pm 0.37$ \\
\hline
\end{tabular}

Table 4. Strong couplings of the $J / \psi$ meson to two charmed mesons: relativistic quark model vs. QCD sum rule.

\begin{tabular}{|c|c|c|c|}
\hline Coupling Approach & Quark model [15-17] & QCD sum rules & References \\
\hline $\begin{array}{l}D-D-J / \psi \\
D-D^{*}-J / \psi\end{array}$ & $\begin{array}{l}26.04 \pm 1.43 \\
(10.7 \pm 0.4) \mathrm{GeV}^{-1}\end{array}$ & $\begin{array}{l}11.6 \pm 1.8 \\
(4.0 \pm 0.6) \mathrm{GeV}^{-1}\end{array}$ & $\begin{array}{l}{[18]} \\
{[18]}\end{array}$ \\
\hline$D_{s^{-}}-D_{s^{-}} J / \psi$ & $23.83 \pm 0.78$ & $11.96_{-1.16}^{+1.34}$ & [19] \\
\hline$D_{s}-D_{s}^{*}-J / \psi$ & $(9.6 \pm 0.8) \mathrm{GeV}^{-1}$ & $\left(4.30_{-1.22}^{+1.53}\right) \mathrm{GeV}^{-1}$ & [20] \\
\hline
\end{tabular}




\section{Acknowledgements}

D. M. would like to express gratitude for support by the Austrian Science Fund (FWF) under project P29028-N27.

\section{References}

[1] P. Santorelli, Phys. Rev. D 77 (2008) 074012, arXiv:hep-ph/0703232.

[2] W. Lucha, F. F. Schöberl, and D. Gromes, Phys. Rep. 200 (1991) 127.

[3] F. Cardarelli, E. Pace, G. Salmè, and S. Simula, Phys. Lett. B 357 (1995) 267, arXiv:nucl-th/ 9507037.

[4] R. N. Faustov and V. O. Galkin, Z. Phys. C 66 (1995) 119.

[5] D. Melikhov and B. Stech, Phys. Rev. D 62 (2000) 014006, arXiv:hep-ph/0001113.

[6] D. Melikhov and B. Stech, Phys. Rev. D 74 (2006) 034022, arXiv:hep-ph/0606203.

[7] W. Lucha, D. Melikhov, and S. Simula, Phys. Rev. D 74 (2006) 054004, arXiv:hep-ph/0606281.

[8] C. T. H. Davies et al., Phys. Rev. D 82 (2010) 114504, arXiv:1008.4018 [hep-lat].

[9] W. Lucha, D. Melikhov, and S. Simula, Phys. Lett. B 701 (2011) 82, arXiv:1101.5986 [hep-ph].

[10] D. Bečirević et al., J. High Energy Phys. 1202 (2012) 042, arXiv:1201.4039 [hep-lat].

[11] G. C. Donald et al. (HPQCD Coll.), Phys. Rev. D 86 (2012) 094501, arXiv:1208.2855 [hep-lat].

[12] W. Lucha, D. Melikhov, and S. Simula, Phys. Lett. B 735 (2014) 12, arXiv:1404.0293 [hep-ph].

[13] K. A. Olive et al. (Particle Data Group), Chin. Phys. C 38 (2014) 090001 and 2015 update.

[14] D. Melikhov, Eur. Phys. J. direct C4 (2002) 2, arXiv:hep-ph/0110087.

[15] W. Lucha, D. Melikhov, H. Sazdjian, and S. Simula, Phys. Rev. D 93 (2016) 016004, 93 (2016) 019902(E), arXiv:1506.09213 [hep-ph].

[16] W. Lucha, D. Melikhov, H. Sazdjian, and S. Simula, Proc. Sci., EPS-HEP 2015 (2015) 428, arXiv:1509.03089 [hep-ph].

[17] W. Lucha, D. Melikhov, H. Sazdjian, and S. Simula, preprint HEPHY-PUB 970/16 (2016), arXiv:1607.05569 [hep-ph].

[18] R. D. Matheus et al., Int. J. Mod. Phys. E 14 (2005) 555.

[19] B. Osório Rodrigues, M. E. Bracco, and M. Chiapparini, Nucl. Phys. A 929 (2014) 143, arXiv: 1309.1637 [hep-ph].

[20] B. Osório Rodrigues et al., Eur. Phys. J. A 51 (2015) 28, arXiv:1501.03088 [hep-ph]. 